\title{
Cyclic Bit Loading for Adaptive OFDM in Narrowband Power Line Communications
}

\author{
Cornelius Kaiser, Norbert Mitschke and Klaus Dostert \\ Institute of Industrial Information Technology (IIIT) \\ Karlsruhe Institute of Technology (KIT) \\ Karlsruhe, Germany \\ Email: \{cornelius.kaiser, norbert.mitschke, klaus.dostert\}@kit.edu
}

\begin{abstract}
This paper considers an adaptive orthogonal frequency-division multiplexing (OFDM) transmission scheme for narrowband (NB) power line communication (PLC). The NB PLC channel is found to show strong cyclostationary characteristics due to the periodic mains voltage. However, conventional systems do not use these time-varying channel features. An improvement of the performance is achieved by exploiting the cyclic properties of the channel. Therefore, adaptive bit and power loading methods are of great interest for PLC. In this work, we will evaluate a cyclic loading method for an OFDM system in the CENELEC A-band. Our analysis is based on noise measurements at three different locations over an observation time of 23 hours. Our contribution includes an OFDM system model comprising both additive noise and channel attenuation. Furthermore, we present a method for limiting the amplitude and power spectral density to comply with narrowband regulations. In conclusion, our proposed method achieves a lower bit error rate at all locations. The improvement reaches up to a $10^{2}$ times lower bit error rate compared to non-adaptive carrier allocation at the same data rate.
\end{abstract}

\section{INTRODUCTION}

Due to its high spectral efficiency and simple channel equalization, orthogonal frequency-division multiplexing (OFDM) gained a lot of interest in power line communication (PLC) research. It is now widely used in narrowband (NB) PLC applications and is implemented in the two most prevalent standards G3 and PRIME [1], [2].

The performance of OFDM can be improved by bit and power loading methods, where each carrier is independently modulated. One major impairment of the NB PLC channel is the strong and time-variant noise. Therefore, a static power and bit allocation can only achieve sub-optimal results.

In the past, bit loading methods have been adopted to NB PLC exploiting the cyclostationary nature of the channel. In [3], an adaptive bit loading method was proposed to NB PLC based on simulated cyclostationary noise. Optimal bit loading for broadband PLC has been studied in [4]. The simulations in this work were based on an linear periodically time-varying (LPTV) channel and an additive white Gaussian noise (AWGN) model, as noise power spectral density (PSD) decays with increasing frequency. In [5], a cyclic bit loading scheme was applied to CENELEC A-band with G3 parameters based on measured noise. All these works have in common that the bit loading methods are greedy algorithms, of which the optimality is mathematically shown in [6]. Methods to reduce the computational complexity and signaling overhead has also been a subject of recent studies [7].

Bit loading for PLC was found to give significant improvement of the bit error rate and throughput compared to a static modulation. However, only little research has been done to quantify the advantage of adaptive bit loading for the NB channel based on measurement data. In this work, we evaluate the benefit of cyclic bit loading for NB PLC in detail. Therefore, we will present a greedy-type bit loading method and discuss modifications to comply with the CENELEC EN 50065-1 norm. We will present quantitative simulation results under real world conditions, based on an extensive set of measured noise and reference channel attenuations.

The remainder of this paper is organized as follows: In section II, we introduce our OFDM system model. In section III, we present our bit loading algorithm in detail. In section IV, we address necessary modifications to our bit loading method to comply with NB regulations. Our measurement setup will be presented in section V. Finally, the paper is concluded by the presentation of our results in section VI.

\section{System Model}

We consider an $N$-point OFDM system with perfect symbol and frequency synchronization. With the sampling frequency $f_{s}$, the discrete spectrum of the OFDM symbol in the frequency domain is

$$
X_{k}=\sum_{i=0}^{N-1} \frac{1}{\Delta f} \delta\left(k-f_{i}\right) \cdot A_{i} e^{j \varphi_{i}},
$$

where $\Delta f=f_{s} / N$ is the subcarrier spacing, $\delta(\cdot)$ the Dirac function and $A_{i} e^{j \varphi_{i}}$ are the symbols of the modulation constellation. The subcarriers $f_{i}$ are orthogonal if $f_{i}=k \Delta f, k \in$ $\{0, \ldots, N-1\}$. Thus, the constellation points of one subcarrier can be mapped independently of any other subcarrier.

In order to obtain realistic values for our OFDM system, we adopt the modulation parameters from the physical layer specifications of the PRIME standard v1.3.6 [2]. The fundamental parameters are summarized in Table I. In contrast to the PRIME standard, we do not use any channel coding or differential modulation schemes for the subcarriers as we are interested in the raw bit performance. Additionally, our system can consider both phase-shift keying (PSK) and quadrature amplitude modulation (QAM) constellation types. 
TABLE I

OFDM PARAMETERS ACCORDING TO THE PRIME STANDARD [2]

\begin{tabular}{|l|l|}
\hline FFT length $N$ & 512 \\
\hline Sample frequency $f_{s}$ & $250 \mathrm{kHz}$ \\
\hline Cyclic prefix (CP) $N_{\mathrm{CP}}$ & 48 samples \\
\hline Data subcarriers & $86-182$ (total 97) \\
\hline Frequency range & $41.7-89.1 \mathrm{kHz}$ \\
\hline Subcarrier spacing $\Delta f$ & $488.3 \mathrm{~Hz}$ \\
\hline Symbol interval $T_{s}$ & $2.24 \mathrm{~ms}$ \\
\hline
\end{tabular}

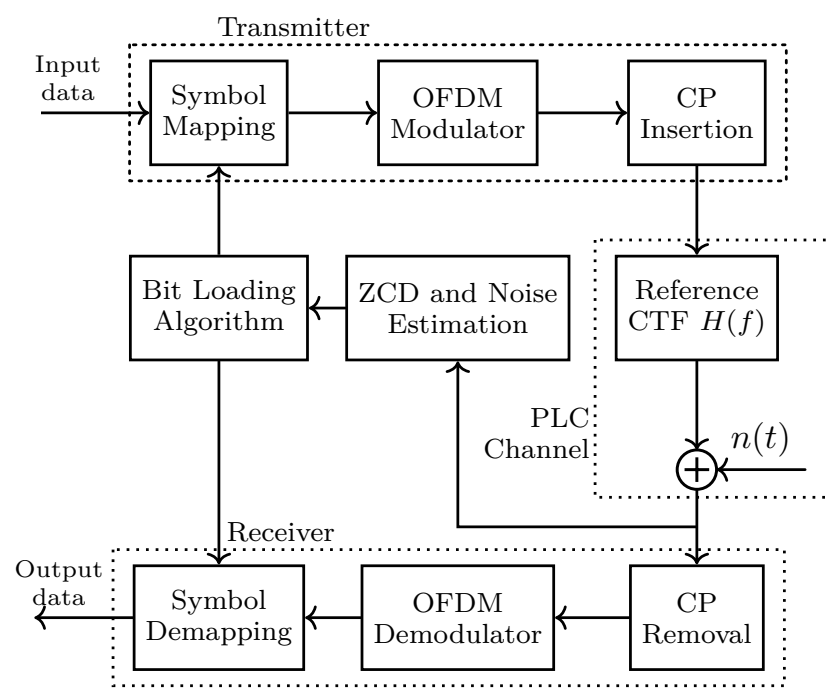

Fig. 1. Adaptive OFDM system model.

In Fig. 1 an complete overview of the system model is given. The transmitter signal is filtered by our channel model and obtained at the receiver. Based on the PSD estimation of the noise $n(t)$ and channel transfer function (CTF) $H(f)$, the bit loading method assigns the symbol mapping at the transmitter and receiver. The $\mathrm{CP}$ is inserted prior to sending the OFDM symbol and removed before the demodulation. A zero crossing detector $(\mathrm{ZCD})$ is used as a timing reference for the noise estimation and the symbol map. We assume that a perfect feedback channel exists and the symbol mapping information is available at the transmitter and receiver.

\section{A. Cyclostationary Channel Model}

For our channel model, we will focus on the characteristics in the CENELEC A-band from 3 to $95 \mathrm{kHz}$. In this frequency range, the channel especially suffers from the harsh and timevariant noise environment. As this noise is severely limiting the performance of NB PLC systems, we evaluate our cyclic bit loading method based on real world noise measurements. To resolve the cyclic nature of the channel into a linear system model, we segment it in both time and frequency domain. As the bit mapping of a single OFDM symbol needs to be constant, the smallest time-domain resolution is $T_{S}$. Within a mains period of $T_{\mathrm{AC}}=20 \mathrm{~ms}$ (in Europe), $M=\left\lfloor T_{\mathrm{AC}} / T_{S}\right\rfloor=8$ time slots are used. The smallest frequency-domain resolution is equal to the subcarrier spacing

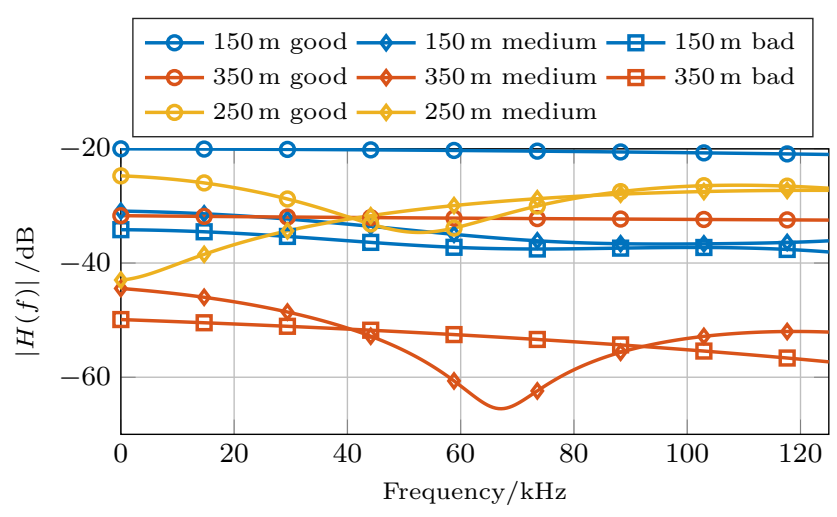

Fig. 2. Reference CTFs for the European low-voltage access domain [8].

$\Delta f$. Accordingly, the number of frequency slots is equal to the number of used subcarriers $K=97$. We will assume that the noise and CTF is flat for each time and frequency slot. Thus, the influence of the channel is completely described in the frequency domain with a linear channel model. For the received OFDM symbols follows

$$
Y_{k}^{(m)}=H_{k} X_{k}^{(m)}+N_{0, k}^{(m)}
$$

where $H_{k}$ is the complex CTF and $N_{0, k}^{(m)}$ is the additive noise of the $m$-th time slot and $k$-th subcarrier. In our system model, the CTF is considered to be stationary. Therefore, the cyclostationary effects are characterized solely by the additive noise. However, the methods presented in this paper can also be applied to a cyclostationary CTF. In this case, the attenuation of the time-variant transfer function can be included in the time-variant noise after channel equalization. By the OPERA project a set of reference CTFs has been defined [8]. These will be used as $H(f)$ to allow for the frequency-dependent attenuation. A subset of the reference channels we used for our evaluation is depicted in Fig. 2.

\section{B. Spectral Density Estimation}

The bit loading method depends on a precise estimation of the noise and CTF. The estimation of the noise is based on $W$ noise samples (with exclusion of the $\mathrm{CP}$ ) at the receiver. Synchronized by the $\mathrm{ZCD}$, the noise signal $\mathbf{n}=$ $[n(1), \ldots, n(W)]$ is rearranged into $L=W / N$ segments, with length $N=T_{s} f_{s}-N_{\mathrm{CP}}$ :

$$
\begin{aligned}
\mathbf{n}^{(m)}(l)= & {[n((l-1) M N+(m-1) N+1), \ldots,} \\
& n((l-1) M N+(m-1) N+N)],
\end{aligned}
$$

where $l$ points to the number of a complete mains cycles and $m$ points to the time slot within the mains period. The concatenated vector $\tilde{\mathbf{n}}^{(m)}=\left[\mathbf{n}^{(m)}(1), \ldots, \mathbf{n}^{(m)}(L)\right]$ with elements $\tilde{n}_{i}^{(m)}$ is used as an input for a PSD estimator. We used the Welch's method with an overlap of $N / 2$ for estimating the PSD for a fixed $m$ :

$$
\hat{N}_{0, k}^{(m)}=\frac{1}{(2 L-1) N} \sum_{l=0}^{2 L-2}\left|\sum_{i=0}^{N-1} \tilde{n}_{i+l N / 2}^{(m)} e^{j 2 \pi \frac{i k}{N}}\right|^{2} .
$$


TABLE II

SYMBOL ENERGY APPROXIMATIONS FOR $M$-PSK AND $M$-QAM ACCORDING TO [12]

\begin{tabular}{|c|c|c|}
\hline$b$ & Name & Symbol energy $E\left(b, N_{0}\right)$ \\
\hline 0 & - & 0 \\
\hline 1 & BPSK & $\frac{N_{0}}{2} \cdot Q^{-1}\left(P_{b}\right)^{2}$ \\
\hline 3 & 8-QAM & $3 N_{0} \cdot Q^{-1}\left(\frac{6 P_{b} b}{5}\right)^{2}$ \\
\hline o/w & $M$-PSK & $\frac{N_{0}}{2} \cdot\left(\frac{Q^{-1}\left(P_{b} b / 2\right)}{\sin \left(\pi / 2^{b}\right)}\right)^{2}$ \\
\hline o/w & $M$-QAM & $\frac{(M-1) N_{0}}{3} \cdot Q^{-1}\left(\sqrt{\frac{\left(1-\sqrt{1-P_{b} b}\right) \cdot \sqrt{M}}{2(\sqrt{M}-1)}}\right)$ \\
\hline
\end{tabular}

\section{Channel Estimation an Equalization}

For estimating the CTF, we use equidistant pilot tones at every 8th subcarrier in a comb-type block structure and a total of 5 OFDM symbols prior to each transmission. In total, 13 out of possible 97 pilot tones are used to increase the power of each pilot subcarrier. The optimal pilot symbols with minimal peak amplitude can then be numerically obtained. With the least squares (LS) channel estimation approach, the coefficients $\hat{H}_{k}$ are obtained as

$$
\hat{H}_{k}=\frac{Y_{k}^{(m)}}{X_{k}^{(m)}}=H_{k}+\Psi_{k}^{(m)}, \text { with } \Psi_{k}^{(m)}=\frac{N_{0, k}^{(m)}}{X_{k}^{(m)}} .
$$

Between the 13 estimated points the CTF needs to be interpolated. This is done by inserting zeros and a subsequent lowpass filtering as described in [9]. With the channel estimate $\hat{H}_{k}$, (2) can be re-written as

$$
Y_{k}^{(m)}=\hat{H}_{k} X_{k}^{(m)}-\Psi_{k}^{(m)} X_{k}^{(m)}+N_{0, k}^{(m)} .
$$

From this equation it can be seen that the channel estimation introduces an additional noise term $\Psi_{k}^{(m)} X_{k}^{(m)}$ at the receiver. This is taken into account with the calculation of the socalled effective noise $N_{0, k \text {,eff }}^{(m)}=-\Psi_{k}^{(m)} X_{k}^{(m)}+N_{0, k}^{(m)}$ [10]. Equalization of the received data is finally done by calculating $\hat{X}_{k}^{(m)}=Y_{k}^{(m)} / \hat{H}_{k}$. For simplicity, we will always refer to the effective noise PSD after channel equalization in the following. Therefore, we will write $\hat{N}_{0, k, \text { eff }}^{(m)} /\left|\hat{H}_{k}\right|^{2}$ in short as $\hat{N}_{0, k}^{(m)}$.

\section{Bit LOADING Method}

The energy and number of bits allocated to each subcarrier of the OFDM system can be chosen independently. In order to achieve the best performance, bit loading methods are used to solve the maximum rate optimization problem $\max \sum_{i=1}^{K} b_{i}$ under the constraint of $\sum_{i=1}^{K} E_{i}=E_{\max }$, where $E_{i}$ is the energy and $b_{i}$ are the bits per symbol (i.e. the allocated bits) of subcarrier $i$. The optimal energy distribution is found by the so-called water-filling method [11].

One drawback of the water-filling method is that an additional rate quantization step is needed as the rates are non-integer

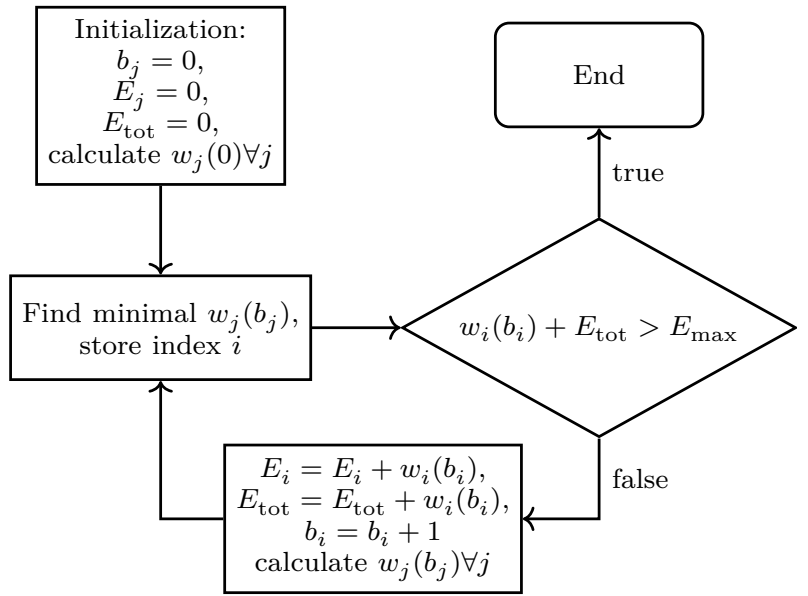

Fig. 3. Flowchart of the greedy algorithm based on [4].

values. As listed in the introduction, discrete rate methods based on greedy algorithms have been developed to overcome this problem. In the following, we will present our bit loading method based on the algorithm by Tunç et al. in [4]. The idea is to increment the rate of the subcarrier that needs the least amount of energy to convey one more bit. This is repeated until all energy is distributed. The energy that is additionally needed to convey one more bit, i.e. $b_{j}+1$ bits on the $j$-th subcarrier, is the differential energy

$$
w_{j}\left(b_{j}\right)=\left\{\begin{array}{l}
E\left(b_{j}+1, \hat{N}_{0, j}\right)-E\left(b_{j}, \hat{N}_{0, j}\right), b_{j}<b_{\max } \\
\infty, \text { otherwise }
\end{array}\right.
$$

with the maximum of $b_{\max }=6$. The energy function can be calculated with approximations as summarized in Table II and the Q-function $Q(x)=1 / \sqrt{2 \pi} \int_{x}^{\infty} \exp \left(u^{2} / 2\right) \mathrm{d} u$. The flowchart of the method is shown in Fig. 3. After the rates and energies have been initialized, $w_{j}(0)$ is calculated for all subcarriers. This corresponds to the differential energy to convey 1 bit. In the following loop, the index of the subcarrier with the smallest $w_{j}\left(b_{j}\right)$ is searched: $i=\arg \min _{j} w_{j}\left(b_{j}\right)$. The maximum energy to be distributed is $E_{\max }$. As long as the differential energy $w_{i}\left(b_{i}\right)$ and the totally assigned energy $E_{\text {tot }}$ is smaller than the maximum energy $E_{\max }, b_{i}$ is increased by 1 and $E_{i}$ and $E_{\text {tot }}$ are updated. Then the loop continues by calculating $w_{j}\left(b_{j}\right)$ for all $j$.

We use a constant target bit error rate (BER) for all subcarriers over time. Thus, the bit loading method is performed independently for each $m$-th time slot within the mains period.

\section{CENELEC Regulatory Aspects}

For the purpose of our simulations we consider the CENELEC regulations given in the norm EN 50065-1 in Europe [13]. For signals with a bandwidth greater than $5 \mathrm{kHz}$, the following regulations apply: 1.) The maximum signal spectrum, measured with peak detector and a bandwidth of $200 \mathrm{~Hz}$, must not exceed $120 \mathrm{~dB} \mu \mathrm{V}$. 2.) The maximum signal amplitude at the transmitter must not exceed $134 \mathrm{~dB} \mu \mathrm{V}$. Both measurements 
need to be performed with a line impedance stabilisation network, which is also specified by the norm.

The first condition is a limitation of the power spectral density. To comply with it, the maximum amplitude per subcarrier is set to $1 \mathrm{~V}$ for simplicity. As the subcarrier bandwidth is larger than the measurement bandwidth, there is still some margin to increase the amplitude. For the maximum subcarrier energy it follows that

$$
E_{\mathrm{SC}, \max }=\frac{N P_{\mathrm{SC}, \max }}{f_{s}}=\frac{1 \mathrm{~V}^{2}}{2} \cdot \frac{N}{f_{s}},
$$

where $N=512$ is the FFT length and $N / f_{s}$ the symbol length without the $\mathrm{CP}$ (see Table I). $P_{\mathrm{SC} \text {,max }}$ is the maximum subcarrier power which is equal to the root mean square of a sine wave with amplitude $1 \mathrm{~V}$.

The second condition can be ensured by limiting the output amplitude to $U_{\max }=5 \mathrm{~V}$. However, clipping the signal leads to unwanted distortion. Therefore, it must be ensured that the error due to clipping can be neglected compared to the error induced by the channel. It is useful to define the clipping ratio $x=U_{\max } / \sigma$, where $\sigma^{2}$ is the average OFDM signal power before clipping. With $x$, the maximum energy to distribute among the subcarriers for the bit loading method is given as

$$
E_{\max }=\frac{N \sigma^{2}}{f_{s}}=\frac{N U_{\max }^{2}}{f_{s} x^{2}} .
$$

With this formula the clipping ratio $x$ can be set as desired. Therefore, our greedy method is modified with the additional test condition $E_{i}+w_{i}>E_{\mathrm{SC} \text {, max }}$ that is inserted in the test block before $w_{i}+E_{\mathrm{tot}}>E_{\max }$. If the test condition holds true, $w_{i}=\infty$ is set and the next minimal $w_{i}$ is tested. If the equation is false, the algorithm continues accordingly.

\section{Measurement Data}

For the data acquisition we used an automated measurement system based on the universal software radio peripheral (USRP) N210. On each of the two USRPs inputs we connected a custom passive coupling unit and a mains voltage ZCD. All measurements were taken over a period of 23 hours on March 3rd and 4th, 2016 in Karlsruhe, Germany. The measurements were taken synchronously at 3 different locations every 2.5 minutes for a span of $10 \mathrm{~s}$. The access points were located at a transformer station (S1), the basement of a house (S2) and a garage (S3). All measurement sites $\mathrm{S} 1-\mathrm{S} 3$ were located on the campus of the Karlsruhe Institute of Technology. In total, the data set consists of 1659 single measurements. The measurement data were collected with a sampling rate of $5 \mathrm{MHz}$. In a pre-processing stage, the measured data are low pass filtered and digitally down-converted to the sampling rate $f_{s}=250 \mathrm{kHz}$ of the simulation.

\section{CyClic Bit LoAding Results}

In the following, we will discuss the results of the cyclic bit loading method under real world conditions. For all simulations, we set the target BER to $10^{-3}$ and used QAM as the modulation type. The ZCD signal was used to extract 450 mains periods out of each measurement signal. The extracted signal was divided into 100 mains periods that were used for noise estimation and 350 that were used for transmission. We set the clipping ratio to 3.67 , which was found leading to good performance by former simulations. Each transmission of 8 OFDM symbols was triggered with the detection of the zero crossing. The remaining $2.08 \mathrm{~ms}$ of the mains period was left unused to compensate for any ZCD jitter.

\section{A. Short-term Analysis}

First of all, we will take a close look at the result of the bit mapping for a single measurement over a complete mains cycle. In Fig. 4 the PSD of the effective noise is depicted together with the symbol mapping output by the bit loading method. The noise was taken from site S3 at 22:00 and the $250 \mathrm{~m}$ medium reference channel was used. In the PSD one can see two fundamental characteristics of the NB noise. Firstly, it shows periodicity in which predominant fundamental cyclic frequency is twice the mains frequency. Therefore, if desired, the method can be easily adjusted using half-cycles of the mains period which also halves the signaling overhead. Secondly, the spectral density is decreasing with increasing frequency. In the bit mapping one can see that the strong noise in the lower frequency range causes the subcarriers to be excluded from the transmission. In the worst case, the lower subcarriers would not be able to attain the target BER with any modulation type. Thus, reducing the overall performance in the case of a static allocation drastically.

\section{B. Long-term Analysis}

We will now discuss the results of the long-term evaluation over the complete $23 \mathrm{~h}$ measurement set. The reference channel was set to ' $250 \mathrm{~m}$ good'. In order to achieve a comparison of the bit loading and static modulation, the bit loading method was executed first. The average number of bits per subcarrier was then truncated and assigned to the static modulation. Additionally, we implemented a feedback noise mitigation based on the methods presented in [15], as impulsive noise has a strong impact on the OFDM performance. The results are shown in Fig. 5. It can be seen, that each measurement site shows a different behavior: At location S1 and S3 the target BER is well achieved with our proposed method. Even while the bit rate is higher with bit loading, it achieves a much better BER compared to the static modulation by 1-2 orders of magnitude.

Location S3 shows the highest noise floor. The lowest possible transmission rate of 1 bit per subcarrier corresponds to a data rate of approx. $38 \mathrm{kbit} / \mathrm{s}$. Even with a 1 bit assignment only a BER $>10^{-2}$ is achieved. Therefore, no reliable communication might be expected in such a scenario. A dependence on the daytime, at which the performance is evaluated, can be observed at location S1. During nighttime a higher bit rate and a lower BER are achieved compared to daytime. This can be attributed to the fact, that the measurement site is located on the university campus with less activity during the night. At location S2, the BER with bit loading fluctuates 

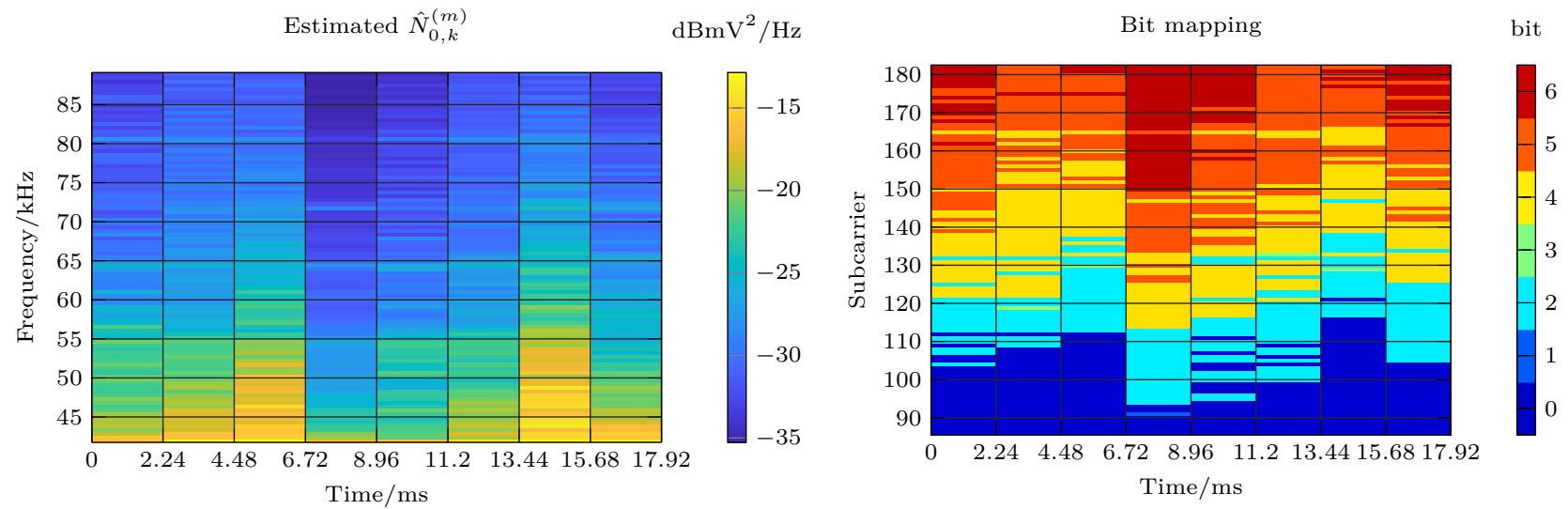

Fig. 4. Cyclic bit mapping corresponding to the effective noise PSD. The measurement was taken at measurement site S2 at 22:00.
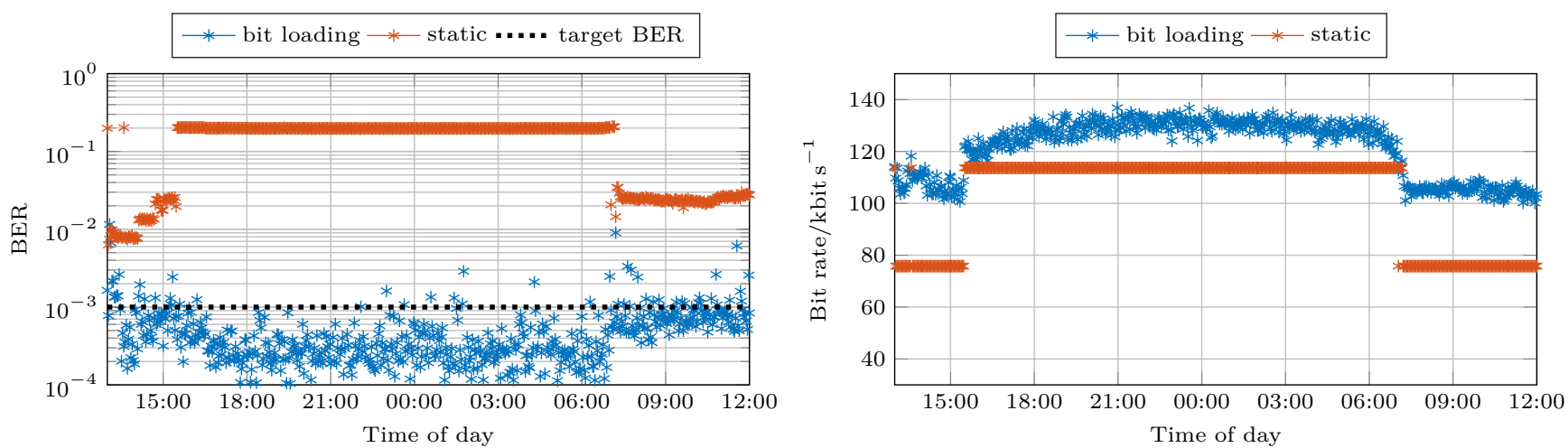

(a) Transformer station $(\mathrm{S} 1)$
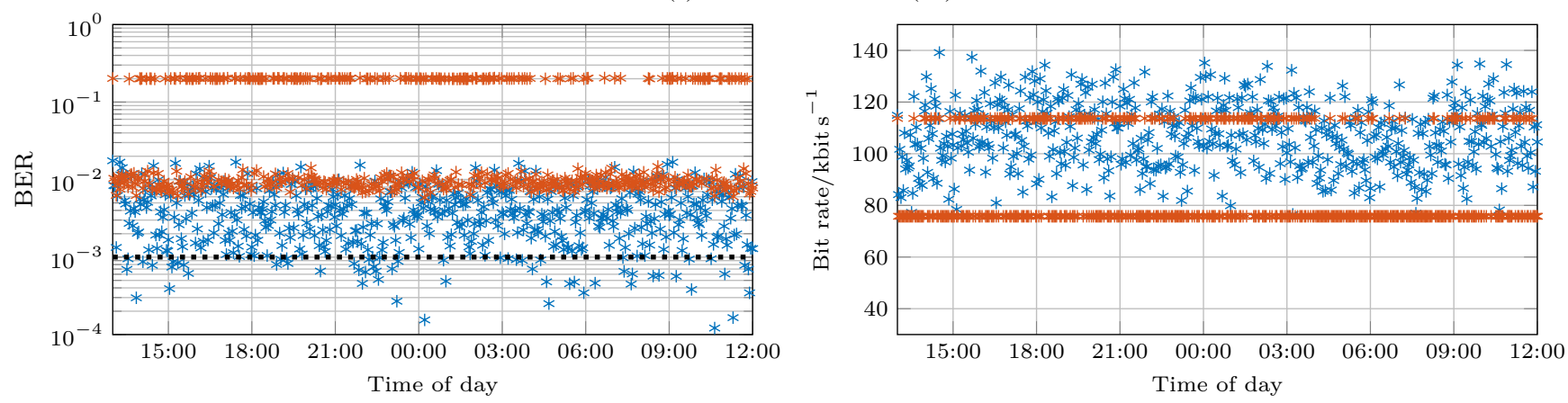

(b) Basement (S2)
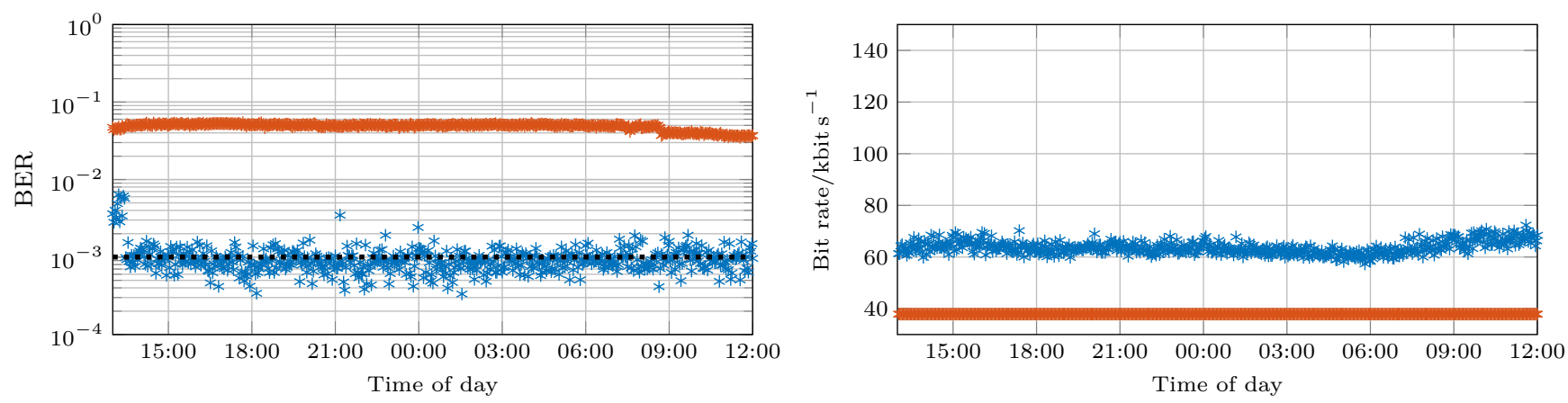

(c) Garage (S3)

Fig. 5. Long-term evaluation of the BER and bit rate over time. The adaptive bit loading method is compared to the static bit allocation. 


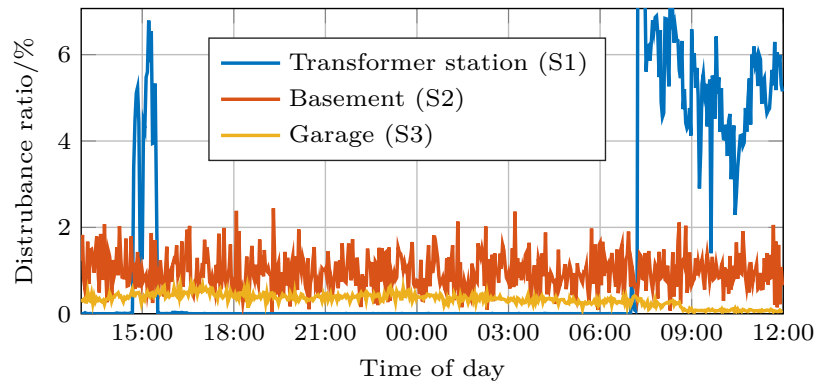

Fig. 6. Disturbance ratio due to impulsive noise during each $10 \mathrm{~s}$ measurement as defined in [14]. The detection threshold was set to $70 \mathrm{mV}$ for location $\mathrm{S} 1$ and $\mathrm{S} 2$ and to $200 \mathrm{mV}$ for location S3.

TABLE III

ACHIEVED AVERAGE BIT RATE AND BER IMPROVEMENTS

\begin{tabular}{c|cc|cc} 
Site & $\left(\text { Rate }_{\text {static }}\right)_{\mathrm{avg}}$ & $\left(\text { Rate }_{\mathrm{BL}}\right)_{\mathrm{avg}}$ & $\left(G_{\text {Rate }}\right)_{\mathrm{avg}}$ & $\left(G_{\mathrm{BER}}\right)_{\mathrm{avg}}$ \\
\hline $\mathrm{S} 1$ & $101.7 \mathrm{kbit} / \mathrm{s}$ & $121.2 \mathrm{kbit} / \mathrm{s}$ & $21.3 \%$ & $23.8 \mathrm{~dB}$ \\
\hline $\mathrm{S} 2$ & $88.1 \mathrm{kbit} / \mathrm{s}$ & $107.1 \mathrm{kbit} / \mathrm{s}$ & $23.9 \%$ & $8.8 \mathrm{~dB}$ \\
\hline $\mathrm{S} 3$ & $37.9 \mathrm{kbit} / \mathrm{s}$ & $63.7 \mathrm{kbit} / \mathrm{s}$ & $68.1 \%$ & $17.2 \mathrm{~dB}$
\end{tabular}

between $10^{-2}$ and $10^{-3}$, together with the data rate which is between $75 \mathrm{kbit} / \mathrm{s}$ and $140 \mathrm{kbit} / \mathrm{s}$. Both can be explained by the strong disturbance due to aperiodic impulses measured at this location. A summary of the disturbance ratio due to impulsive noise is shown for all locations in Fig. 6. If strong aperiodic noise occurs during the channel estimation, the bit rate is lower which tends to result in less errors. If no or only few aperiodic impulses are encountered during the channel estimation the bit rate can be too optimistic, which results in the target BER not being achieved.

To summarize the long-term observations, we calculated the sample mean and sample variance of the BER and data rate gain. We define the relative gain of the BER in $\mathrm{dB}$ as

$$
G_{\mathrm{BER}}=10 \log _{10}\left(\frac{\mathrm{BER}_{\text {static }}}{\mathrm{BER}_{\mathrm{BL}}}\right),
$$

where the index BL refers to the bit loading case. The relative gain of the data rate is defined as follows

$$
G_{\text {Rate }}=\left(\frac{\text { Rate }_{\text {BL }}-\text { Rate }_{\text {static }}}{\text { Rate }_{\text {static }}}\right) .
$$

The results are shown in Table III. On average, there was an improvement of the BER by $8 \mathrm{~dB}$ to $23 \mathrm{~dB}$. Furthermore, the bit rate was improved by $21 \%$ to $68 \%$. The greatest improvement was achieved at the locations S2 and S3, where the noise showed strong cyclostationarity. At location S2, which suffered most from the impulsive noise, the target BER was not attained. However, the data rate and BER were still improved compared to a static allocation.

\section{CONCLUSIONS}

In this work, we have evaluated a cyclic bit loading method for NB PLC using real world measurements. We presented our greedy-type algorithm as well as the modifications needed to comply with regulatory limits of the amplitude and power spectral density. We applied our method to a set of 1659 individual measurements and calculated the improvement on the BER and bit rate. Our results show that both the BER and bit rate were improved in all scenarios. Furthermore, our cyclic bit loading method enabled a reliable transmission in conditions where no communication would be possible with a static bit allocation.

\section{REFERENCES}

[1] Narrowband orthogonal frequency division multiplexing power line communication transceivers for G3-PLC networks, Recommendation ITU-T G.9903, International Telecommunication Union, Aug. 2017.

[2] Draft specification for powerline intelligent metering evolution R.1.3.6, PRIME Alliance Technical Working Group. [Online]. Available: http: //www . prime - alliance . org/wp - content/uploads/2013/04/PRIMESpec_v1.3.6.pdf.

[3] N. Sawada, T. Yamazato, and M. Katayama, "Bit and power allocation for power-line communications under nonwhite and cyclostationary noise environment," in IEEE International Symposium on Power Line Communications and Its Applications, Mar. 2009, pp. 307-312.

[4] M. A. Tunc, E. Perrins, and L. Lampe, "Optimal LPTV-aware bit loading in broadband PLC,' IEEE Transactions on Communications, vol. 61 , no. 12 , pp. 5152-5162, Dec. 2013.

[5] K. Nieman, J. Lin, M. Nassar, K. Waheed, and B. Evans, "Cyclic spectral analysis of power line noise in the $3-200 \mathrm{kHz}$ band," in IEEE International Symposium on Power Line Communications and Its Applications, Mar. 2013, pp. 315-320.

[6] E. Baccarelli and M. Biagi, "Optimal integer bit-loading for multicarrier ADSL systems subject to spectral-compatibility limits," Signal Processing, vol. 84, no. 4, pp. 729-741, 2004.

[7] G. R. Colen, L. G. de Oliveira, A. J. H. Vinck, and M. V. Ribeiro, "A spectral compressive resource allocation technique for PLC systems," IEEE Transactions on Communications, vol. 65, no. 2, pp. 816-826, Feb. 2017.

[8] K. D. M. Babic M. Hagenau and J. Bausch, Theoretical postulation of PLC channel models, 2005, Deliverable 'D4' within the OPERA IST Integrated Project No. 507667, Funded by EC.

[9] S. Coleri, M. Ergen, A. Puri, and A. Bahai, "Channel estimation techniques based on pilot arrangement in OFDM systems," IEEE Transactions on Broadcasting, vol. 48, no. 3, pp. 223-229, Sep. 2002.

[10] C. Liu and R. Mathar, "Optimal and efficient bit loading for OFDM in the presence of channel uncertainty," in IEEE International Symposium on Wireless Communication Systems, Oct. 2008, pp. 11-15.

[11] Y. G. Li, Ed., Orthogonal frequency division multiplexing for wireless communications. New York, NY: Springer, 2006.

[12] J. G. Proakis and M. Salehi, Digital communications. McGraw-Hill, 2009.

[13] Signaling on low-voltage electrical installations in the frequency range $3 \mathrm{khz}$ to 148,5 khz - part 1: General requirements, frequency bands and electromagnetic disturbances, CENELEC Standard EN 50065-1, European Committee for Electrotechnical Standardization, Apr. 2011.

[14] M. Zimmermann and K. Dostert, "Analysis and modeling of impulsive noise in broad-band powerline communications," IEEE Transactions on Electromagnetic Compatibility, vol. 44, no. 1, pp. 249-258, Feb. 2002.

[15] A. Mengi and A. J. H. Vinck, "Successive impulsive noise suppression in OFDM," in IEEE International Symposium on Power Line Communications and Its Applications, Mar. 2010, pp. 33-37. 
Karlsruher Institut für Technologie

\section{Repository KITopen}

Dies ist ein Postprint/begutachtetes Manuskript.

\section{Empfohlene Zitierung:}

Kaiser, C.; Mitschke, N.; Dostert, K.

Cyclic bit loading for adaptive OFDM in narrowband power line communications. 2018. 2018 IEEE International Symposium on Power Line Communications and its Applications, ISPLC 2018; Manchester; United Kingdom; 8 April 2018 through 11 April 2018. doi: $\underline{10.5445 / I R / 1000083952}$

Zitierung der Originalveröffentlichung:

Kaiser, C.; Mitschke, N.; Dostert, K. Cyclic bit loading for adaptive OFDM in narrowband power line communications. 2018. 2018 IEEE International Symposium on Power Line Communications and its Applications, ISPLC 2018; Manchester; United Kingdom; 8 April 2018 through 11 April 2018, Institute of Electrical and Electronics Engineers (IEEE).

doi:10.1109/ISPLC.2018.8360225 